\title{
A low complexity user grouping based multiuser MISO downlink precoder
}

Saif Khan Mohammed and Erik G. Larsson

Linköping University Pre Print

N.B.: When citing this work, cite the original article.

(C2011 IEEE. Personal use of this material is permitted. However, permission to reprint/republish this material for advertising or promotional purposes or for creating new collective works for resale or redistribution to servers or lists, or to reuse any copyrighted component of this work in other works must be obtained from the IEEE.

Saif Khan Mohammed and Erik G. Larsson, A low complexity user grouping based multiuser MISO downlink precoder, 2011, accepted for IEEE GLOBECOM 2011.

Preprint available at: Linköping University Electronic Press

http://urn.kb.se/resolve?urn=urn:nbn:se:liu:diva-70205 


\title{
A low complexity user grouping based multiuser MISO downlink precoder
}

\author{
Saif Khan Mohammed, Member IEEE and Erik G. Larsson, Senior Member IEEE \\ Communication Systems Division, Dept. Electrical Engineering (ISY), \\ Linköping University, Sweden. E-mail: saif@isy.liu.se and erik.larsson@isy.liu.se.
}

\begin{abstract}
We consider low complexity precoding for the Multiple Input Single Output (MISO) Gaussian Broadcast channel with $N_{t}$ antennas at the base station and $N_{u}$ single antenna users in the downlink. Theoretical studies have suggested high throughput communication with increasing spatial dimensions i.e., $\min \left(N_{t}, N_{u}\right)$. Nevertheless, most modern communication standards are unable to exploit the spatial dimension fully, since they are restricted to orthogonal communication techniques like TDMA/FDMA (Time/Frequency Division Multiplexed Access) which are known to be sub-optimal. This restriction is mostly due to the prohibitive complexity of optimal/near-optimal precoding schemes. On the other hand low complexity techniques like Zero Forcing (ZF) and MMSE have poor sum rate performance. In this paper, we propose a novel low-complexity user grouping based precoding scheme which schedules all users on the same time-frequency resource (i.e., optimal utilization of resources). The proposed precoder is analytically shown to achieve a sum rate performance significantly better than the $\mathrm{ZF}$ precoder at similar complexity. Through simulations, it is also observed to achieve a significant fraction of the sum rate achieved by the optimal schemes.
\end{abstract}

\section{INTRODUCTION}

Multiple-Input Multiple-Output (MIMO) technology holds the key to very high throughput downlink communication in fading wireless channels by exploiting the spatial dimension [1]. However most modern wireless communication standards support a maximum achievable spectral efficiency less than $10 \mathrm{bits} / \mathrm{sec} / \mathrm{Hz}$. This is because the multiple access scheme is still TDMA or FDMA, in which each user communicates over distinct frequency-time resource, i.e., orthogonal communication. The rate region and sum capacity of the Gaussian MIMO broadcast channel (which models downlink communication in modern wireless systems) is achieved by a scheme called Dirty Paper Coding (DPC), in which all users share the same frequency-time resource [2]. It is also known that orthogonal access schemes (like TDMA, FDMA) are strictly sub-optimal and achieve only a small fraction of the total sum capacity [3]. However, TDMA and FDMA are still favored in practice, due to less burden of obtaining channel state information and also due to the high precoding complexity of optimal precoders like DPC. Other near-optimal precoders like those based on vector perturbation and lattice reduction [4] also have prohibitive complexity. On the other hand low complexity precoders, like ZF [5], MMSE are are known to achieve poor sum rate performance especially in ill-conditioned channels.

This work was supported by the Swedish Foundation for Strategic Research (SSF) and ELLIIT.
To keep the low-complexity benefit of the ZF precoder and yet improve the overall sum rate, we propose a user grouping based precoder. In the proposed precoder, the users are divided into small groups of equal size. Downlink beamforming is done in such a way that, at each receiver the interference from the signal intended for users not in its group is nulled out. However, there still remains interference from the signal of users in the same group. This interference is pre-cancelled at the transmitter, by performing dirty paper coding among the users in the same group. With small groups (having only 2 or 3 users), dirty paper coding within each group is practically feasible and is equivalent to dirty paper coding for a MISO broadcast channel with small number of users [6], [7].

Inter-group interference pre-cancellation for a group of users is achieved by choosing their beamforming vectors to lie in a space orthogonal to the space spanned by the channel vectors of the users in the other groups. One novel aspect of the proposed precoder, is that we choose the beamforming vectors in such a way that the effective channel matrix for each group is lower triangular, which enables successive known interference pre-cancellation within each group using DPC. With group size greater than one and a per user power allocation same as that of the ZF precoder, the proposed precoder is analytically shown to achieve a sum rate greater than that achieved by the ZF precoder. For a given grouping of users, the optimal power allocation is given by the waterfilling scheme. Since the achievable sum rate is observed to be sensitive towards the chosen grouping of users, it is jointly optimized w.r.t. both the per user power allocation as well as the grouping of users. This optimization problem is inherently complex, and therefore we propose near-optimal low-complexity solutions to it. Through analysis and simulations, we show that indeed the proposed user grouping based precoder achieves a sum rate significantly greater than that achieved by the ZF precoder ${ }^{2}$, at similar complexity. The low complexity attribute of the proposed precoder could be an enabler for large MISO broadcast systems with large $N_{t}$ and $N_{u}$.

We also clarify that, the proposed precoder is entirely dif-

\footnotetext{
${ }^{2}$ In this paper, the $\mathrm{ZF}$ precoder used as the benchmark precoder for comparison, is based on the pseudo-inverse of the channel matrix, which is one among all possible generalized inverses. In [8], it has been shown that with a total transmit power constraint, among all possible generalized inverses, the pseudo-inverse results in the maximum achievable sum rate. Since we only consider a total transmit power constraint in this paper, comparison with the pseudo-inverse based ZF precoder suffices and therefore we need not compare with ZF precoder based on other generalized inverses.
} 
ferent from the block diagonalization based precoder proposed in [5], which considers a MIMO multiuser broadcast channel, in which each user could have multiple receive antennas. Beamforming vectors are chosen such that each user sees no interference from the information intended for other users. Hence, in the special case of MISO broadcast channel (which we consider in this paper), the block diagonalization precoder in [5] basically reduces to the ZF precoder. In addition to this, the precoder that we propose performs beamforming in groups of users and not separately for each user. Another user pairing precoder has been proposed for the Gaussian multiuser MIMO broadcast channel in [9]. However, in [9], only 2 users share the same time-frequency resource, i.e., the medium access is orthogonal in groups of 2 users, which is a sub-optimal utilization of resources when compared to the proposed precoder where all users share the same timefrequency resource.

Notations: $\mathbf{A}^{H}$ and $\mathbf{A}^{T}$ represent conjugate transpose and transpose of the matrix $\mathbf{A}$ respectively. For any arbitrary complex number $z$, let $\bar{z}$ and $|z|$ denote its complex conjugate and absolute value respectively. The complex and the real fields are denoted by $\mathbb{C}$ and $\mathbb{R}$ respectively. Given a vector $\mathbf{x}=\left(x_{1}, x_{2}, \cdots, x_{n}\right)^{T} \in \mathbb{C}^{n}$, let $\|x\| \triangleq \sqrt{\sum_{k=1}^{n}\left|x_{k}\right|^{2}}$. For any positive integer $n, n ! \triangleq n$. $(n-1) .(n-2) . \cdots .2 .1$. Further, for any set $S,|S|$ denotes the cardinality (size) of the set $S$.

\section{SYSTEM MODEL}

Let $\mathbf{H}=\left(\mathbf{h}_{1}, \mathbf{h}_{2}, \cdots, \mathbf{h}_{N_{u}}\right)^{H}$, represent the $N_{u} \times N_{t}$ channel matrix between the base station and the $N_{u}$ users $^{3}$ $\left(N_{t} \geq N_{u}\right)$. The channel vector from the transmitter to the $k$-th user is denoted by $\mathbf{h}_{k}^{H} \in \mathbb{C}^{1 \times N_{t}}$, with its $i$-th entry $\bar{h}_{k, i}$ representing the channel gain from the $i$-th transmit antenna to the receive antenna of the $k$-th user ${ }^{4}$. With channel knowledge at the transmitter, the information symbols can be effectively mapped onto the symbols to be transmitted from the $N_{t}$ transmit antennas. Let $\mathbf{x}=\left(x_{1}, x_{2}, \cdots, x_{N_{t}}\right)^{T} \in \mathbb{C}^{N_{t} \times 1}$ represent the transmitted vector. The vector of received symbols $\mathbf{y}=\left(y_{1}, y_{2}, \cdots, y_{N_{u}}\right)^{T} \in \mathbb{C}^{N_{u} \times 1}$ (with $y_{k}$ denoting the signal received by the $k$-th user) is then given by

$$
\mathbf{y}=\mathbf{H} \mathbf{x}+\mathbf{n}
$$

where $\mathbf{n}=\left(n_{1}, n_{2}, \cdots n_{N_{u}}\right)^{T} \in \mathbb{C}^{N_{u} \times 1}$ is the additive noise vector with $n_{k}$ representing the noise at the $k$-th receiver. Further, each entry of $\mathbf{n}$ is an i.i.d $\mathbb{C N}(0,1)$ random variable. Further, the transmitter is subject to an average transmit power constraint given by

$$
\mathbb{E}\left[\|\mathbf{x}\|^{2}\right]=P_{T} .
$$

Due to unit variance noise, we would refer to $P_{T}$ as the transmit signal to receiver noise ratio (i.e., transmit SNR).

For the sake of clarity and conciseness we introduce the following notations. Subsequently we shall refer to the $k$-th

\footnotetext{
${ }^{3}$ Throughout the paper, $\mathbf{H}$ is assumed to be full rank.

${ }^{4}$ Subsequently we shall also refer to the receiver at the $k$-th user as the $k$-th receiver.
}

user by $\mathcal{U}_{k}$. In the proposed precoding scheme, the total set of users $\mathcal{S}=\left\{\mathcal{U}_{1}, \mathcal{U}_{2}, \cdots \mathcal{U}_{N_{u}}\right\}$ is partitioned into $N_{g}=N_{u} / g$ disjoint groups of $\operatorname{size}^{5} \mathrm{~g}$. Let the $i$-th group of users be denoted by the ordered set $\mathcal{S}_{i}=\left\{\mathcal{U}_{i_{1}}, \mathcal{U}_{i_{2}}, \cdots, \mathcal{U}_{i_{g}}\right\}$. Therefore, $\mathcal{S}=\cup_{i=1}^{N_{g}} \mathcal{S}_{i}$, and $\mathcal{S}_{i} \cap \mathcal{S}_{j}=\phi, \forall i \neq j$, where $\phi$ denotes the null set. Also, let any arbitrary grouping of users be denoted by the unordered set $\mathcal{P}=\left\{\mathcal{S}_{1}, \mathcal{S}_{2}, \cdots, \mathcal{S}_{N_{g}}\right\}$. For example, with $N_{u}=4$ and $g=2$, one possible grouping of users is given by $\mathcal{P}=\left\{\left\{\mathcal{U}_{1}, \mathcal{U}_{4}\right\},\left\{\mathcal{U}_{2}, \mathcal{U}_{3}\right\}\right\}$.

For notational purposes, let us denote the set of all possible groupings of a set of $N_{u}$ users into groups of size $g$, by $\mathcal{A}_{N_{u}}^{(g)}$. For example with $N_{u}=4$ users and $g=2$

$$
\begin{aligned}
\mathcal{A}_{4}^{(2)}= & \left\{\left\{\left\{\mathcal{U}_{1}, \mathcal{U}_{2}\right\},\left\{\mathcal{U}_{3}, \mathcal{U}_{4}\right\}\right\},\left\{\left\{\mathcal{U}_{2}, \mathcal{U}_{1}\right\},\left\{\mathcal{U}_{3}, \mathcal{U}_{4}\right\}\right\},\left\{\left\{\mathcal{U}_{1}, \mathcal{U}_{2}\right\},\left\{\mathcal{U}_{4}, \mathcal{U}_{3}\right\}\right\},\right. \\
& \left\{\left\{\mathcal{U}_{2}, \mathcal{U}_{1}\right\},\left\{\mathcal{U}_{4}, \mathcal{U}_{3}\right\}\right\},\left\{\left\{\mathcal{U}_{1}, \mathcal{U}_{3}\right\},\left\{\mathcal{U}_{2}, \mathcal{U}_{4}\right\}\right\},\left\{\left\{\mathcal{U}_{3}, \mathcal{U}_{1}\right\},\left\{\mathcal{U}_{2}, \mathcal{U}_{4}\right\}\right\}, \\
& \left\{\left\{\mathcal{U}_{1}, \mathcal{U}_{3}\right\},\left\{\mathcal{U}_{4}, \mathcal{U}_{2}\right\}\right\},\left\{\left\{\mathcal{U}_{3}, \mathcal{U}_{1}\right\},\left\{\mathcal{U}_{4}, \mathcal{U}_{2}\right\}\right\},\left\{\left\{\mathcal{U}_{1}, \mathcal{U}_{4}\right\},\left\{\mathcal{U}_{3}, \mathcal{U}_{2}\right\}\right\}, \\
& \left.\left\{\left\{\mathcal{U}_{4}, \mathcal{U}_{1}\right\},\left\{\mathcal{U}_{3}, \mathcal{U}_{2}\right\}\right\},\left\{\left\{\mathcal{U}_{1}, \mathcal{U}_{4}\right\},\left\{\mathcal{U}_{2}, \mathcal{U}_{3}\right\}\right\},\left\{\left\{\mathcal{U}_{4}, \mathcal{U}_{1}\right\},\left\{\mathcal{U}_{2}, \mathcal{U}_{3}\right\}\right\}\right\} .
\end{aligned}
$$

Let $\mathbf{H}[i] \in \mathbb{C}^{\left(N_{u}-g\right) \times N_{t}}$ denote the sub-matrix of $\mathbf{H}$ consisting of only those rows which represent the channel vector of users not in the set $\mathcal{S}_{i}$, and let $\mathbf{G}[i] \in \mathbb{C}^{g \times N_{t}}$ denote the sub-matrix containing the remaining rows of $\mathbf{H}$. Specifically, if $\mathcal{S}_{i}=\left\{\mathcal{U}_{i_{1}}, \mathcal{U}_{i_{2}}, \cdots, \mathcal{U}_{i_{g}}\right\}$ then

$$
\mathbf{G}[i] \triangleq\left(\mathbf{h}_{i_{1}}, \mathbf{h}_{i_{2}}, \cdots, \mathbf{h}_{i_{g}}\right)^{H} .
$$

Further let $\mathcal{H}_{i}$ represent the subspace spanned by the rows of $\mathbf{H}[i]$, and let $\mathcal{H}_{i}^{\perp}$ be the subspace orthogonal to $\mathcal{H}_{i}$. Therefore,

$$
\mathbf{P}[i]=\left(I_{N_{t}}-\mathbf{H}[i]^{H}\left(\mathbf{H}[i] \mathbf{H}[i]^{H}\right)^{-1} \mathbf{H}[i]\right) \in \mathbb{C}^{N_{t} \times N_{t}}
$$

represents the projection matrix for the subspace $\mathcal{H}_{i}^{\perp}$.

\section{ZF PRECODER AND THE MOTIVATION FOR GROUPING USERS}

One of the most simple and low complexity linear precoder is the ZF precoder. For each user, the ZF precoder beamforms the user's information is a direction which is orthogonal to the space spanned by the channel vectors of the remaining $N_{u}-1$ users, resulting in no inter-user interference. Further, for any given user, its effective channel gain is proportional to the Euclidean length of the projection of its channel vector onto the space orthogonal to the space spanned by the channel vectors of remaining users. In case of ill-conditioned channels, since the channel vectors of all the users are "highly" linearly dependent, the effective channel gain of each user would be small, implying low achievable rates. It would therefore be ideal to keep the low-complexity benefit of the ZF precoder and yet improve the overall sum rate, especially when the channel is ill-conditioned.

By grouping users into groups of size larger than one, beamforming can be done to nullify only inter-group interference. With small group size, intra-group interference can then be pre-cancelled using practical DPC at the transmitter, without any significant increase in the required transmit power.

\footnotetext{
${ }^{5}$ The proposed precoder can be generalized to have groups of different size. However, for simplicity, we only consider groups of equal size in this paper.
} 
Therefore the effective channel gain for $\mathcal{U}_{i_{j}}$ is the Euclidean length of the projection of $\mathbf{h}_{i_{j}}^{H}$ onto the space $\mathcal{H}_{i}^{\perp}$. On the other hand, with the ZF precoder, the effective channel gain is the Euclidean length of the projection of $\mathbf{h}_{i}^{H}$ onto the subspace orthogonal to all the rows of $\mathbf{H}$ except $\mathbf{h}_{i_{j}}^{H}$ (We shall subsequently denote this orthogonal subspace by $\mathcal{H}_{i_{j}}^{\perp}$ ). It is noted that $\mathcal{H}_{i_{j}}^{\perp} \subset \mathcal{H}_{i}^{\perp}$ whenever $g>1$. Since the projection of a vector onto a subspace of some space $\mathcal{G}$ is of lesser Euclidean length than its projection onto the space $\mathcal{G}$, it follows that the effective channel gain for $\mathcal{U}_{i_{j}}$ is higher with the proposed user grouping based precoder as compared to that with the ZF precoder. This simple observation coupled with the availability of practical low-complexity DPC for small systems, motivates the proposed precoding scheme which is presented in the next section in more detail.

\section{Proposed User Grouping based Precoder}

For the $i$-th group of users, let the QR decomposition of the matrix $\mathbf{F}[i] \triangleq \mathbf{P}[i] \mathbf{G}[i]^{H}$ be given by

$$
\mathbf{F}[i]=\mathbf{Q}[i] \mathbf{R}[i]
$$

where $\mathbf{R}[i] \in \mathbb{C}^{g \times g}$ is an upper triangular matrix with positive diagonal entries, and $\mathbf{Q}[i] \in \mathbb{C}^{N_{t} \times g}$ is a matrix with orthonormal columns. From the above decomposition it is also clear that, the $g$ columns of $\mathbf{Q}[i]$ form an orthonormal basis for the space $\mathcal{H}_{i}^{\perp}$. Further, for any $k \neq i$, we have

$$
\mathbf{G}[k] \mathbf{Q}[i]=\mathbf{0}, k \neq i .
$$

This is because, for any $k \neq i$, the rows of $\mathbf{G}[k]$ lie in $\mathcal{H}_{i}$.

Beamforming the information for the users in the $i$-th group along the columns of $\mathbf{Q}[i]$ ensures that users in group $i$ do not observe any interference from other groups. The precoding operation for the $i$-th group is therefore given by

$$
\mathbf{x}[i]=\mathbf{Q}[i] \mathbf{W}[i] \mathbf{u}[i]
$$

where $\mathbf{u}[i] \triangleq\left(u_{i_{1}}, u_{i_{2}}, \cdots, u_{i_{g}}\right)^{T}$ is the $g \times 1$ vector of auxiliary input symbols of the users in the $i$-th group $\mathcal{S}_{i}$. Information is encoded over these auxiliary input symbols. The auxiliary symbols are assumed to be i.i.d. Gaussian distributed with mean 0 and variance 1 . $\mathbf{W}[i] \in \mathbb{C}^{g \times g}$ is an additional linear precoder to optimize the sum rate achieved by the $i$-th group of users. The transmitted vector is then given by

$$
\mathbf{x}=\sum_{i=1}^{N_{g}} \mathbf{x}[i] .
$$

Let $\mathbf{y}[i] \triangleq\left(y_{i_{1}}, y_{i_{2}}, \cdots, y_{i_{g}}\right)^{T}$ be the $g \times 1$ vector of symbols received by the users in the $i$-th group $\mathcal{S}_{i}$. Using (1), (7) and (8), the received vector $\mathbf{y}[i]$ is given by

$$
\begin{aligned}
\mathbf{y}[i] & =\mathbf{G}[i]\left(\mathbf{x}[i]+\sum_{k=1, k \neq i}^{N_{g}} \mathbf{x}[k]\right)+\mathbf{n}[i] \\
& =\mathbf{G}[i] \mathbf{x}[i]+\sum_{k=1, k \neq i}^{N_{g}} \mathbf{G}[i] \mathbf{Q}[k] \mathbf{W}[k] \mathbf{u}[k]+\mathbf{n}[i] \\
& =\mathbf{G}[i] \mathbf{x}[i]+\mathbf{n}[i]=\mathbf{G}[i] \mathbf{Q}[i] \mathbf{W}[i] \mathbf{u}[i]+\mathbf{n}[i]
\end{aligned}
$$

where the last step follows from the application of (6). From (9) it is clear that each group of users does not have any interference from the other groups. Basically the original MISO broadcast channel has been decomposed into $N_{g}$ parallel noninterfering $g$-user MISO broadcast subchannels.

We next focus on the effective channel matrix for the $i$-th group of users. From (9) it is again clear that the effective channel matrix for the $i$-th group of users is given by

$$
\mathbf{B}[i] \triangleq \mathbf{G}[i] \mathbf{Q}[i] \mathbf{W}[i] .
$$

In this paper, we restrict ourselves to diagonal $\mathbf{W}[i]=$ $\operatorname{diag}\left(\sqrt{p_{i_{1}}}, \sqrt{p_{i_{2}}}, \cdots \sqrt{p_{i_{g}}}\right)$, where $p_{i_{j}}$ is the power allocated to the information symbol of the $j$-th user in the $i$-th group. With diagonal $\mathbf{W}[i]$, the sum power constraint in (2) is

$$
\sum_{i=1}^{N_{g}} \sum_{j=1}^{g} p_{i_{j}}=P_{T} .
$$

Subsequently, let $\mathbf{p}=\left(p_{1}, p_{2}, \cdots, p_{N_{u}}\right)$ denote the power allocation vector, with $p_{i}$ being the power allocated to $\mathcal{U}_{i}$.

We next show that $\mathbf{B}[i]$ is actually a lower triangular matrix and is equal to $\mathbf{R}[i]^{H} \mathbf{W}[i]$. From the definitions of $\mathbf{P}[i]$ and $\mathbf{Q}[i]$ in (4) and (5), it is clear that $\mathbf{P}[i]$ is the projection matrix for the space spanned by the columns of $\mathbf{Q}[i]$ and therefore

$$
\mathbf{P}[i] \mathbf{Q}[i]=\mathbf{Q}[i] .
$$

Since $\mathbf{F}[i]=\mathbf{Q}[i] \mathbf{R}[i]=\mathbf{P}[i] \mathbf{G}[i]^{H}$ it follows that $\mathbf{R}[i]=$ $\mathbf{Q}[i]^{H} \mathbf{P}[i] \mathbf{G}[i]^{H}$, and hence using (12) and the fact that $\mathbf{P}[i]$ is Hermitian, we have

$$
\mathbf{Q}[i]^{H} \mathbf{G}[i]^{H}=\mathbf{R}[i] .
$$

Combining (13) with (9) we have

$$
\mathbf{y}[i]=\mathbf{R}[i]^{H} \mathbf{W}[i] \mathbf{u}[i]+\mathbf{n}[i] .
$$

From (14), the received signal at the $j$-th user in the $i$-th group is given by

$$
\begin{aligned}
& y_{i_{j}}=\mathbf{R}[i]_{(j, j)} \sqrt{p_{i_{j}}} u_{i_{j}}+(\overbrace{\left(\sum_{k=1}^{j-1)} \overline{\mathbf{R}[i]_{(k, j)} \sqrt{p_{i_{k}}}} u_{i_{k}}\right.}^{\text {Interference term }}+n_{i_{j}}, \\
& j=1,2, \cdots g
\end{aligned}
$$

where $\mathbf{R}[i]_{(k, j)}$ denotes the entry of $\mathbf{R}[i]$ in the $k$-th row and the $j$-th column. Due to the lower triangular structure of the effective channel matrix for the $i$-th group, from (15), we observe that the $j$-th user in the $i$-th group (i.e., $\mathcal{U}_{i_{j}}$ ) has interference only from the symbols of the previous $(j-1)$ users in the same group (i.e., $\mathcal{U}_{i_{1}}, \cdots \mathcal{U}_{i_{(j-1)}}$ ).

In the proposed coding scheme, we start with the first user in the $i$-th group, and since it sees no interference from any other users, we simply use an AWGN channel code with rate

$$
r_{i_{1}}=\log _{2}\left(1+p_{i_{1}} \mathbf{R}[i]_{(1,1)}^{2}\right)
$$

The second user, has an interference term with contribution only from the first user. Since the transmitter knows the transmitted symbol for the first user, it knows the interference 
term for the second user, and can therefore perform known interference pre-cancellation using the Dirty Paper Coding scheme [10], [11], [12]. In a similar manner, for the $j$-th user, the transmitter can perform Dirty Paper Coding for the known interference term which has contributions only from the previous $(j-1)$ users. The rate achieved by the $j$-th user in the $i$-th group is therefore given by

$$
r_{i_{j}}=\log _{2}\left(1+p_{i_{j}} \mathbf{R}[i]_{(j, j)}^{2}\right) .
$$

For a given grouping of users $\mathcal{P} \in \mathcal{A}_{N_{u}}^{(g)}$, total power constraint $P_{T}$, channel realization $\mathbf{H}$ and power allocation vector $\mathbf{p}$, the sum rate achieved is given by

$$
r\left(\mathbf{H}, P_{T}, \mathcal{P}, \mathbf{p}\right) \triangleq \sum_{i=1}^{N_{u} / g} \sum_{j=1}^{g} r_{i_{j}}
$$

where $r_{i_{j}}$ is given by (17). Maximization over $\mathbf{p}$ yields

$$
r\left(\mathbf{H}, P_{T}, \mathcal{P}\right) \triangleq \max _{\mathbf{p} \mid \sum_{i=1}^{N_{u}} p_{i}=P_{T}, p_{i} \geq 0} r\left(\mathbf{H}, P_{T}, \mathcal{P}, \mathbf{p}\right)(19)
$$

In (19), the optimal power allocation for a given grouping of users is given by the waterfilling scheme [13]. Subsequently, for $g=1$, we shall denote the optimal waterfilling power allocation in (19), by $\mathbf{p}^{*}=\left(p_{1}^{*}, p_{2}^{*}, \cdots, p_{N_{u}}^{*}\right)$. The optimal sum rate is achieved by jointly maximizing $r\left(\mathbf{H}, P_{T}, \mathcal{P}, \mathbf{p}\right)$ over both $\mathcal{P}$ and $\mathbf{p}$ and is given by

$$
\begin{aligned}
C\left(\mathbf{H}, P_{T}\right) & =\underset{\mathcal{P} \in \mathcal{A}_{N_{u}}^{(g)}, \mathbf{p} \mid \sum_{i=1}^{N_{u}} p_{i}=P_{T}}{\max } r\left(\mathbf{H}, P_{T}, \mathcal{P}, \mathbf{p}\right) \\
& =\max _{\mathcal{P} \in \mathcal{A}_{N_{u}}^{(g)}} r\left(\mathbf{H}, P_{T}, \mathcal{P}\right) .
\end{aligned}
$$

This optimization problem is inherently complex due to its combinatorial nature. It has been observed that the achievable sum rate is sensitive to the chosen grouping of users. This observation therefore motivates us to consider optimal/nearoptimal methods for solving (20).

For small $N_{u}$, (20) can be solved simply by brute-force enumeration of all possible groupings. However, for large $N_{u}$, the combinatorial nature of the problem makes it inherently complex to solve by brute-force enumeration. Towards this end, in Section $\mathrm{V}$ we propose low-complexity approximations to the solution of (20).

Here we also note that, the $\mathrm{ZF}$ precoder is a special case of the proposed user grouping scheme with $g=1$, i.e., $N_{u}$ groups with one user per group. The other special case is for $g=N_{u}$, i.e., only one group consisting of all the $N_{u}$ users. We shall refer to this as the ZF-DPC precoding scheme and has been discussed in detail in [11] as the ZF-DP precoder. Note that with $g=N_{u}$, successive DPC has to be performed for $N_{u}$ users, which can be prohibitive for large $N_{u}$. Further, the number of possible ordered groupings is $N_{u}$ ! which is also large for large $N_{u}$.

\section{PARTITIONING USERS INTO GROUPS}

In this section, we consider the optimization problem in (20) and propose low-complexity near-optimal solutions to it. We firstly show that irrespective of the channel realization $\mathbf{H}$, the sum rate achieved by the proposed precoder with any arbitrary grouping $(g \geq 2)$ and the $\mathrm{ZF}$ power allocation, is greater than that achieved with $g=1$.

Theorem 1: Let $\mathcal{P} \in \mathcal{A}_{N_{u}}^{g}=\left\{\mathcal{S}_{1}, \mathcal{S}_{2}, \cdots, \mathcal{S}_{N_{g}}\right\}, \mathcal{S}_{k}=$ $\left\{\mathcal{U}_{k_{1}}, \mathcal{U}_{k_{2}}, \cdots, \mathcal{U}_{k_{g}}\right\}$ be any arbitrary user grouping with $g>$ 1. Then

$$
r\left(\mathbf{H}, P_{T}, \mathcal{P}, \mathbf{p}^{*}\right)=\sum_{k=1}^{N_{u} / g} \sum_{j=1}^{g} \log _{2}\left(1+p_{k_{j}}^{*} \mathbf{R}[k]_{(j, j)}^{2}\right) \geq C_{\mathrm{ZF}} .
$$

is satisfied for any channel realization $\mathbf{H} . C_{\mathrm{ZF}}$ is the sum rate achieved by the $\mathrm{ZF}$ precoder.

We are unable to present the proof here due to lack of space. We note that Theorem 1 holds for any arbitrary user grouping. Therefore optimizing $r\left(\mathbf{H}, P_{T}, \mathcal{P}, \mathbf{p}^{*}\right)$ w.r.t. $\mathcal{P}$ is expected to achieve a sum rate significantly greater than $C_{\mathrm{ZF}}$. We therefore propose the following optimization problem.

$$
\mathcal{P}^{*}=\arg \max _{\mathcal{P} \in \mathcal{A}_{N_{u}}^{g}} \sum_{k=1}^{N_{u} / g} \sum_{j=1}^{g} \log _{2}\left(1+p_{k_{j}}^{*} \mathbf{R}[k]_{(j, j)}^{2}\right) .
$$

In the next section, we propose a low-complexity near-optimal solution to the problem in (22).

\section{A. Generalized User Grouping Algorithm - GUGA}

For a given $\mathbf{H}$ and $P_{T}$, the optimization problem in (22) can be shown to be equivalent to a weighted matching problem (WMP) on $g$-uniform hypergraphs. This correspondence can be used to solve (22). However, the general WMP is known to be NP-hard, and is therefore not realizable in polynomial time. Hence several polynomial time approximation algorithms have been proposed, using standard linear and semidefinite programming techniques [14]. However even these approximations are too complex for application to symbol by symbol precoding. We therefore propose a low-complexity (polynomial time) user grouping algorithm to the optimization problem in (22), which is shown to achieve a sum rate greater than $1 / g$ of the sum rate achieved with the optimal grouping $\mathcal{P}=\mathcal{P}^{*}\left(\mathcal{P}^{*} \text { is given by }(22)\right)^{6}$.

Before discussing the algorithm in detail, we define the weight of any given group of users as follows. Given the $k$-th group of $g$ users, represented by the ordered set $\mathcal{S}_{k}=$ $\left\{\mathcal{U}_{k_{1}}, \mathcal{U}_{k_{2}}, \cdots, \mathcal{U}_{k_{g}}\right\}$, we define its weight to be ${ }^{7}$

$$
\mathcal{W}\left(\mathcal{S}_{k}\right) \triangleq \sum_{j=1}^{g} \log _{2}\left(1+p_{k_{j}}^{*} \mathbf{R}[k]_{(j, j)}^{2}\right) .
$$

The optimization problem in (22) is therefore expressed in terms of the weight function as

$$
\mathcal{P}^{*}=\arg \max _{\mathcal{P} \in \mathcal{A}_{N_{u}}^{g}} \sum_{k=1}^{N_{u} / g} \mathcal{W}\left(\mathcal{S}_{k}\right) .
$$

\footnotetext{
${ }^{6}$ Since, $g$ is typically small (usually $2,3,4$ ), $1 / g$ is a significant fraction of 1.

${ }^{7}$ We reminder the reader that $\mathbf{R}[k]$ is implicitly dependent on the chosen grouping.
} 
The proposed algorithm is an iterative greedy algorithm. Let the current set of active users after the $k$-th iteration be denoted by $\mathbb{V}^{(k)} \subset \mathcal{S}$. In the $(k+1)$-th iteration, a subset of $\mathbb{V}^{(k)}$ containing $g$ users is chosen to be the $k$-th group of users for the proposed algorithm. Let $\mathbb{E}^{(k)}$ denote the set of all possible ordered subsets of $\mathbb{V}^{(k)}$ of size $g$. That is

$$
\mathbb{E}^{(k)} \triangleq\left\{s \subset \mathbb{V}^{(k)}|| s \mid=g\right\} .
$$

Starting with the $k=0$-th iteration the set $\mathbb{V}^{(0)}=\mathcal{S}$ (i.e., all users are active) and $\mathbb{E}^{(0)}$ is the set of all possible ordered subsets of $\mathcal{S}$ of size $g$. In the $(k+1)$-th iteration, the proposed algorithm finds the group of $g$-users in $\mathbb{E}^{(k)}$ having the maximum weight. This is the $(k+1)$-th group of users for the proposed algorithm and is given by

$$
\tilde{\mathcal{S}}_{k+1}=\left\{\mathcal{U}_{k+1_{1}}, \mathcal{U}_{k+1_{2}}, \cdots, \mathcal{U}_{k+1_{g}}\right\} \triangleq \arg \max _{e \in \mathbb{E}^{(k)}} \mathcal{W}(e) .
$$

where the weight function $\mathcal{W}($.$) is given by (23). Let$ $\mathcal{T}^{(k+1)} \subset \mathbb{E}^{(k)}$ be the set of groups of size $g$ having at least one user in the set $\tilde{\mathcal{S}}_{k+1}$. That is

$$
\mathcal{T}^{(k+1)} \triangleq\left\{e \mid e \in \mathbb{E}^{(k)} \text { and } \mathcal{U}_{k+1_{j}} \in e \text { for some } j\right\}
$$

where $\mathcal{U}_{k+1_{j}}$ is the $j$-th user in the ordered set $\tilde{\mathcal{S}}_{k+1}$. After the $(k+1)$-th iteration, the users $\mathcal{U}_{k+1_{j}}, j=1,2, \cdots, g$ are removed from the active set of users and therefore

$$
\mathbb{V}^{(k+1)}=\mathbb{V}^{(k)}-\tilde{\mathcal{S}}_{k+1} .
$$

It is also clear that $\mathbb{E}^{(k+1)}=\mathbb{E}^{(k)}-\mathcal{T}^{(k+1)}$. The algorithm then moves on to the $(k+2)$-th iteration. Since there are totally $N_{u}$ users and therefore $N_{u} / g$ groups, it is evident that the algorithm terminates after the $N_{g}=\left(N_{u} / g\right)$-th iteration. The grouping of users is then given by

$$
\tilde{\mathcal{P}}=\left\{\tilde{\mathcal{S}}_{1}, \tilde{\mathcal{S}}_{2}, \cdots, \tilde{\mathcal{S}}_{N_{g}}\right\}
$$

We next show that the achievable sum rate with the proposed user grouping $\tilde{\mathcal{P}}$ is lower bounded by $1 / g$ of the sum rate achieved with the optimal grouping $\mathcal{P}^{*}$.

Theorem 2: For any $g \geq 2$, the proposed low-complexity user grouping $\tilde{\mathcal{P}}$ satisfies

$$
r\left(\mathbf{H}, P_{T}, \mathcal{P}^{*}, \mathbf{p}^{*}\right) \geq r\left(\mathbf{H}, P_{T}, \tilde{\mathcal{P}}, \mathbf{p}^{*}\right) \geq \frac{1}{g} r\left(\mathbf{H}, P_{T}, \mathcal{P}^{*}, \mathbf{p}^{*}\right)
$$

We are unable to present the proof due to space constraints. Since the ZF power allocation $\mathbf{p}^{*}$ is not the optimal power allocation for the proposed grouping $\tilde{\mathcal{P}}$, further increase in the sum rate can be achieved by the optimal waterfilling power allocation, which we denote by $\hat{\mathbf{p}}=\left(\hat{p}_{1}, \hat{p}_{2}, \cdots, \hat{p}_{N_{u}}\right)$. Since $\hat{p}_{i}$ is the optimal power allocation for the $i$-th user and not $p_{i}^{*}$,

$$
r\left(\mathbf{H}, P_{T}, \tilde{\mathcal{P}}, \mathbf{p}^{*}\right) \leq r\left(\mathbf{H}, P_{T}, \tilde{\mathcal{P}}, \hat{\mathbf{p}}\right)
$$

Combining (30) and (31) we have

$$
r\left(\mathbf{H}, P_{T}, \tilde{\mathcal{P}}, \hat{\mathbf{p}}\right) \geq \frac{1}{g} r\left(\mathbf{H}, P_{T}, \mathcal{P}^{*}, \mathbf{p}^{*}\right) .
$$

Even though $(\tilde{\mathcal{P}}, \hat{\mathbf{p}})$ is not the optimal solution to (20), it still achieves a significantly better sum rate when compared to the ZF precoder (see Section VI). Further increase in sum rate is possible by fixing the power allocation to $\hat{\mathbf{p}}$ and then computing the user grouping using the proposed GUGA algorithm. We can then fix this as the new user grouping and then compute the new waterfilling power allocation. By repeating this alternating optimization technique, it would be possible to achieve a local optimum to problem (20).

It can be shown that the total precoding complexity is $O\left(g ! g^{3} N_{u}^{g}\right)+O\left(g ! g N_{u}^{(g+1)}\right)+O\left(N_{u}^{2} N_{t}\right)$ plus performing dirty paper coding for $N_{u} / g g$-user MISO broadcast systems. Further for $g=2$, the complexity of the proposed precoder is the same as that of the ZF precoder (i.e., $O\left(N_{u}^{3}\right)$ ).

Remark : For the special case of $g=2$, there exists polynomial time algorithms for WMP [15], which can be used to solve (22) with a complexity of $O\left(N_{u}^{3}\right)$. However, actual implementations of these optimal algorithms rely on the manipulation of complex data structures like heaps. This results in a large coefficient for $N_{u}^{3}$ in the polynomial expression for the run time complexity as compared to the coefficient for the greedy algorithm proposed by us.

\section{Simulation RESUlts}

In this section, we report the sum rates achieved by the proposed user grouping based precoding scheme. Perfect channel state information is assumed at the transmitter. Each receiver is also assumed to have perfect knowledge of its equivalent channel from the transmitter. For a given grouping of users, power allocation is given by the waterfilling scheme, as is discussed in (19). The proposed user grouping is given by (29). For the sake of comparison we also present suitable upper and lower bounds on the DPC sum capacity of the MISO broadcast channel.

An upper bound is simply the capacity of a point-to-point single user $N_{t} \times N_{u}$ MIMO system (i.e., $N_{t}$ transmit and $N_{u}$ receive antennas) with perfect channel state information at both the transmitter and receiver [1]. A tight lower bound on the sum capacity of the Gaussian MISO broadcast channel is given by considering the MAC-BC duality, and is given by [16]

$$
C_{l b}=\log _{2}\left|\mathbf{I}_{N_{t}}+\frac{P_{T}}{N_{u}} \mathbf{H}^{H} \mathbf{H}\right| .
$$

We subsequently assume a $N_{t}=N_{u}$ Gaussian MISO broadcast channel with i.i.d. Rayleigh flat fading statistics. For the sake of comparison, we also plot the sum rates achieved by the ZF precoder (proposed precoder with $g=1$ ) and by the ZF-DPC precoder (proposed precoder with $g=N_{u}$ and the ordered user grouping given by $\left\{\left\{1,2, \cdots, N_{u}\right\}\right\}$ ).

In Fig.1, we report the achievable ergodic sum rate (i,e., averaged over the channel fading statistics) of the proposed user grouping based precoding scheme for a $N_{t}=N_{u}=12$ Gaussian MISO broadcast channel. It is observed that the sum rate achieved by the proposed user grouping scheme with $g=2$, is greater than the sum rate achieved by the ZF precoder. This also implies that the proposed user grouping 


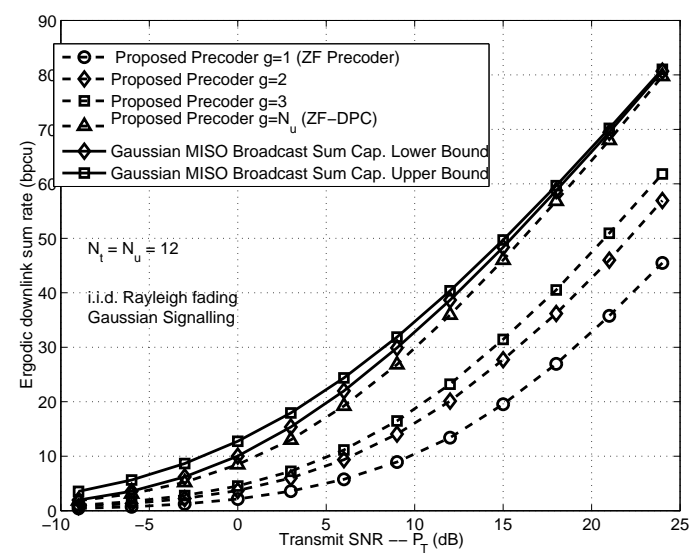

Fig. 1. Ergodic sum rates achieved by the proposed precoder for a $N_{t}=$ $N_{u}=12$ MISO broadcast channel. User grouping for $g=2,3$ is given by the GUGA algorithm.

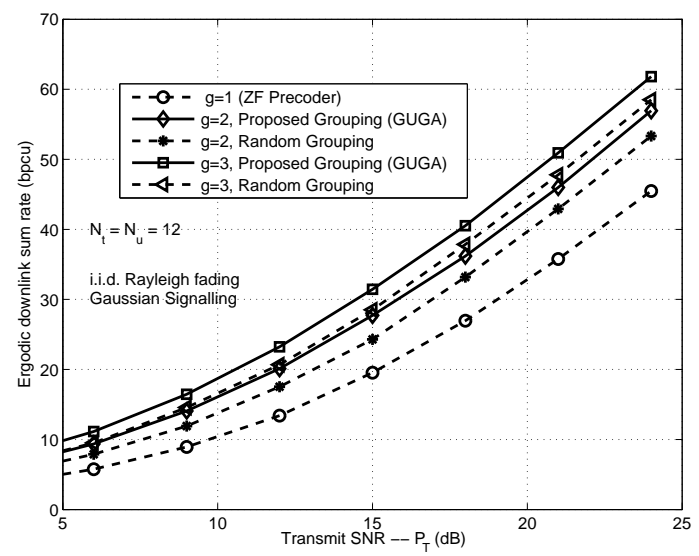

Fig. 2. Ergodic sum rates achieved by the proposed precoder for a $N_{t}=$ $N_{u}=12$ MISO broadcast channel with random grouping and the proposed grouping.

based precoder is more power efficient than the ZF precoder. It is also observed that with increase in the group size the proposed precoder becomes increasingly power efficient. For example, to achieve a sum rate of 30 bits per channel use (bpcu), the transmit SNR required by the proposed precoder with $g=3$ is about $1.2 \mathrm{~dB}$ less than the SNR required with $g=2$. In fact with $g=N_{u}$ (ZF-DPC scheme), the SNR required to achieve a given ergodic sum rate is within $1.0 \mathrm{~dB}$ of the theoretical minimum SNR required to achieve that sum rate in a Gaussian MISO broadcast channel. Also, this implies that the achievable sum rate with the proposed precoder is much larger than $r\left(\mathbf{H}, P_{T}, \mathcal{P}^{*}, \mathbf{p}^{*}\right) / g$. However, the improvement in performance with increasing $g$, comes at the cost of additional complexity. In Fig. 2 we compare the ergodic sum rate achieved by the proposed precoder with random user grouping to that achieved by the proposed user grouping (i.e., $\tilde{\mathcal{P}}$ ) for a $N_{t}=N_{u}=12$ Gaussian MISO broadcast channel. Random user grouping is achieved by grouping the users in any arbitrary manner independent of the channel knowledge. It is observed that, even random grouping results in significant increase in the achievable sum rate when compared to the ZF precoder. This also supports the claims made in Theorem 1. It is also observed that, for the proposed precoding scheme, the proposed user grouping algorithms are more power efficient than random user grouping by about 1.0 $\mathrm{dB}$ at high transmit SNR.

\section{CONCLUSION}

In this paper, we have considered low-complexity precoding for the MISO Gaussian Broadcast channel. We propose a novel user grouping based precoding scheme, which is analytically shown to achieve significantly better sum rates compared to the ZF precoder, at similar complexity. Better performance is achieved compared to the ZF precoder, since multi-user interference-cancellation via linear beamforming is performed only at the group level, which results in effective per-group channel matrices with larger singular values. With small sized groups ( $g=2,3$ ), practical DPC is then performed for known interference pre-cancellation within each group.

\section{REFERENCES}

[1] I. E. Telatar, "Capacity of Multi-antenna Gaussian Channels," European Trans. on Telecommunications, pp. 585-595, vol. 10, no. 6, Dec. 1999.

[2] H. Weingarten, Y. Steinberg, S. Shamai, "The Capacity Region of the Gaussian Multiple-Input Multiple-Output Broadcast Channel," IEEE Trans. on Information Theory, pp. 3936-3964, vol. 52, no. 9, Sept. 2006.

[3] N. Jindal, and A. Goldsmith, "Dirty-paper coding versus TDMA for MIMO Broadcast channels," IEEE Trans. on Information Theory, pp. 1783-1794, vol. 51, no. 5, May 2005.

[4] C. B. Peel, B. M. Hochwald, and A. L. Swindlehurst, "A vector-perturbation technique for near-capacity multiantenna multiuser communication-part I: channel inversion and regularization," IEEE Trans. on Communications, pp. 195-202, vol. 53, no. 1, Jan. 2005.

[5] Q. H. Spencer, A. L. Swindlehurst, and M. Haardt, "Zero-Forcing Methods for Downlink Spatial Multiplexing in Multiuser MIMO Channels," IEEE Trans. on Signal processing, pp. 461-471, vol. 52, no. 2, Feb. 2004.

[6] Y. Sun, Y. Yang, A. Liveris, V. Stankovic and Z. Xiong, "Near Capacity dirty-paper code design : A source channel coding approach," IEEE. Trans. on Information Theory, pp. 3013-3031, vol. 55, no. 7, July 2009.

[7] G. Shilpa, A. Thangaraj, and S. Bhashyam, "Dirty paper coding using sign-bit shaping and LDPC codes," Proc. IEEE International Symposium on Information Theory (ISIT'2010), pp. 923-927, Austin, Texas, June 13-18 2010.

[8] A. Wiesel, Y. C. Eldar, S. Shamai, "Zero-forcing precoding and Generalized inverses," IEEE. Trans. on Signal Proc., pp. 4409-4418, vol. 56, no. 9 , Sept. 2008.

[9] A. Hottinen, and E. Viterbo, "Optimal user pairing in downlink MUMIMO with transmit precoding," 6th IEEE International Symposium on Modeling and Optimization in Mobile, Ad Hoc, and Wireless Networks, WiOPT'2008, pp. 97-99, Berlin, Germany, 1 - 3 April 2008.

[10] M. Costa, "Writing on dirty paper," IEEE. Trans. on Information Theory, pp. 439-441, vol. IT-29, May 1983.

[11] G. Caire and S. Shamai, "On the achievable throughput of a multiantenna Gaussian broadcast channel," IEEE. Trans. on Information Theory, pp. 1691-1706, vol. 49, no. 7, July 2003.

[12] U. Erez, S. Shamai and R. Zamir, "Capacity and lattice-strategies for canceling known interference," IEEE. Trans. on Information Theory, pp. 3820-3833, vol. 51, no. 11, Nov. 2005.

[13] T.M. Cover and Joy A. Thomas, Elements of information theory, John Wiley and Sons, 2nd Ed., July 2006.

[14] Y. H. Chan and L. C. Lau, "On linear and semi-definite programming relaxations for Hypergraph matching," Proc. ACM-SIAM symposium on Discrete Algorithms, (SODA'2010), pp. 1500-1511, Jan. 17-19 2010.

[15] H. N. Gabow, "Data structures for weighted matching and nearest common ancestors with linking," Proc. First annual ACM-SIAM symposium on Discrete Algorithms, (SODA'90), pp. 434-443, 1990.

[16] S. Vishwanath, N. Jindal, A. Goldsmith, "Duality, achievable rates and sum-rate capacity of Gaussian MIMO broadcast channels," IEEE Trans. on Information Theory, pp. 2658-2668, vol. 49, no. 10, Oct. 2003. 